EP-204

\title{
Gravity dependent and hand held 'push' injection method of contrast cholangiography: A pilot study comparing outcomes and efficacy
}

\author{
Neha BHATT ${ }^{1}$, Nalini Kanta GHOSH ${ }^{2}$, Rajan SAXENA ${ }^{* 3}$ \\ 'Department of Surgical Gastroenterology, Center of Hepatobiliary Diseases \& Transplantation, \\ Sanjay Gandhi Post Graduate Institute of Medical Sciences, Lucknow, India \\ ${ }^{2}$ Department of Surgical Gastroenterology, Center of Hepatobiliary Diseases \& Transplantation, \\ Sanjay Gandhi Post Graduate Institute of Medical Sciences, Lucknow, India \\ ${ }^{3}$ Department of Surgical Gastroenterology, Center of Hepatobiliary Diseases \& Transplantation, \\ Sanjay Gandhi Post Graduate Institute of Medical Sciences, Lucknow, India
}

Introduction: Contrast cholangiograms still continue to be obtained prior to removal of intraoperatively or preoperatively placed intra-biliary tubes to look for filling defects, anastomotic leaks or stenosis, evaluation of radiological intervention or a controlled external biliary fistula. Post-cholangiogram cholangitis remains a major morbidity concern. Is it related to the technique?

Methods: An observational prospective pilot study comparing hand held push injection (PIC) and gravity dependent (GDC) techniques of cholangiography in 30 consecutive patients with in-situ biliary tubes after administration of pre-procedure antibiotic, excluding patients with active cholangitis or complete biliary obstruction. In GDC group, dye was delivered under gravity from a height of $25 \mathrm{~cm}$ instead of injection. Outcomes analysed included adverse reactions - minor (two or more of low grade fever, tachycardia, pain/nausea without fever, subclinical cholangitis) and major (two or more of high grade fever, chills, tachycardia, hypotension, oliguria), readmission requirement, and the efficacy (opacification of the biliary tree and demonstration of the objective).

Results: In the PIC group ( $\mathrm{n}=14)$, adverse reactions were minor in $6(42.8 \%)$ and major in $4(28.5 \%)$. Two patients required re-admission, and in two others discharge from the hospital was delayed. In the GDC group ( $\mathrm{n}=16)$, adverse reactions were minor in 7 (43.7\%), with no major adverse reaction or readmission. Major adverse reactions were significantly more common in PIC as compared to GDC $(28.5 \%$ vs. $0 \%$ respectively, $p$-value $=0.03)$, and translated into higher treatment costs.

Conclusions: When required, GDC, limiting rise in intrabiliary pressure, is a safer, completely efficacious and economical technique. 